\title{
Global Routine Vaccination Coverage, 2015
}

\author{
Rebecca M. Casey, MBBS ${ }^{1,2}$; Laure Dumolard, PhD³; M. Carolina Danovaro-Holliday, MD³; Marta Gacic-Dobo, MSc ${ }^{3}$; Mamadou S. Diallo, PhD ${ }^{4}$;
} Lee M. Hampton, $\mathrm{MD}^{2}$; Aaron S. Wallace, $\mathrm{MPH}^{2}$

In 1974, the World Health Organization (WHO) established the Expanded Program on Immunization* to provide protection against six vaccine-preventable diseases through routine infant immunization (1). Based on 2015 WHO and United Nations Children's Fund (UNICEF) estimates, global coverage with the third dose of diphtheria-tetanus-pertussis vaccine (DTP3), the first dose of measles-containing vaccine (MCV1) and the third dose of polio vaccine ( $\mathrm{Pol} 3$ ) has remained stable (84\%-86\%) since 2010. From 2014 to 2015, estimated global coverage with the second MCV dose (MCV2) increased from $39 \%$ to $43 \%$ by the end of the second year of life and from $58 \%$ to $61 \%$ when older age groups were included. Global coverage was higher in 2015 than 2010 for newer or underused vaccines, including rotavirus vaccine, pneumococcal conjugate vaccine $(\mathrm{PCV})$, rubella vaccine, Haemophilus influenzae type $\mathrm{b}(\mathrm{Hib})$ vaccine, and 3 doses of hepatitis B (HepB3) vaccine. Coverage estimates varied widely by WHO Region, country, and district; in addition, for the vaccines evaluated (MCV, DTP3, Pol3, HepB3, Hib3), wide disparities were found in coverage by country income classification. Improvements in equity of access are necessary to reach and sustain higher coverage and increase protection from vaccine-preventable diseases for all persons.

WHO and UNICEF derive national coverage estimates through an annual country-by-country review of all available data, including administrative ${ }^{\dagger}$ and survey-based ${ }^{\S}$ reviews $(2,3)$. To analyze equity of vaccination coverage, countries were categorized by World Bank income classification (low, lower-middle, upper-middle, high) based on 2015 per capita gross national income (GNI) (4) and eligibility for financial support from Gavi, the Vaccine Alliance (Gavi), for new vaccine introduction at any time since 2005 (5). Eligibility for Gavi support is typically based on a country's GNI per capita; the threshold for support started at US $\$ 1,000$ per capita in 2000 and increased to US $\$ 1,580$ by 2016 .

\footnotetext{
*The original EPI vaccines were Bacille Calmette-Guérin vaccine (BCG) (to protect against tuberculosis), polio vaccine ( $\mathrm{Pol})$, measles-containing vaccine (MCV), and diphtheria-tetanus-pertussis vaccine (DTP).

$\dagger$ Administrative coverage is calculated as the number of vaccine doses administered to persons in a specified target group divided by the estimated target population.

$\$$ During vaccination coverage surveys, a representative sample of households are visited and caregivers of children in a specified target group (aged 12-23 months) are interviewed. Dates of vaccination are transcribed from the child's homebased record or are recorded based on caregiver recall or from health facility records. Coverage is calculated as the proportion of persons in a target age group who received a vaccine dose.

Gavi Phase 2 classification (73 eligible countries).
}

Endorsed by the World Health Assembly in 2012, the Global Vaccine Action Plan 2011-2020 (6) calls on all countries to reach $\geq 90 \%$ national coverage and $\geq 80 \%$ coverage in every district or equivalent administrative unit for all vaccines in the country's national immunization schedule by 2020 . DTP3 coverage by age 12 months is an indicator of immunization program performance (2). During 2015, a total of 116 million children received DTP3; DTP3 coverage ranged from $76 \%$ in the WHO African Region to $94 \%$ in the Western Pacific Region (Table 1). National DTP3 coverage estimates ranged from $16 \%$ to $99 \%$ and the national DTP1 (first dose of DTP) to DTP3 dropout rates (the proportion of children who received DTP1 but did not receive DTP3) ranged from $0 \%$ to $61 \%$. Overall, 124 (65\%) countries achieved $\geq 90 \%$ national DTP3 coverage (Table 2). National DTP3 coverage was $80 \%-89 \%$ in 34 countries, $70 \%-79 \%$ in 12 countries, and $<70 \%$ in 22 countries. Among the 19.4 million children worldwide who did not receive 3 DTP doses during the first year of life, 11.7 million (60\%) lived in 10 countries (Figure). Among all children who did not complete the 3-dose DTP series, 12.8 million (66\%) never received the first DTP dose, and 6.6 million (34\%) started, but did not complete the series.

DTP3 coverage estimates in low-income countries were lower than in higher-income countries (Table 2). On average, DTP3 coverage was 11\% lower in countries eligible for 2016 Gavi support (see supplemental figure at https://stacks.cdc. gov/view/cdc/42377). More than half of the 10 countries with the lowest 2015 DTP3 coverage estimates (Equatorial Guinea [16\%]; Ukraine [23\%]; South Sudan [31\%]; Syrian Arab Republic [41\%]; Somalia [42\%]; Central African Republic [47\%]; Guinea [51\%]; Liberia [52\%]; Chad [55\%]; and Nigeria [56\%]) are currently experiencing civil unrest or economic turmoil, which can lead to disruption of vaccination services (7). Complete subnational coverage data, based on country-reported administrative sources, were available for 158 countries in 2015; among these, 54 (34\%) reported achieving $\geq 80 \%$ DTP3 coverage in every district, and $21(13 \%)$ reported that $\geq 10 \%$ of districts had DTP3 coverage $<50 \%$.

MCV1 coverage in 2015 ranged from 74\% in the African Region to $96 \%$ in the Western Pacific Region (Table 1) and from $20 \%$ to $99 \%$ by country; MCV2 coverage varied from $18 \%$ to $93 \%$ by region and from $8 \%$ to $99 \%$ by country. Globally, 119 (61\%) countries achieved the $\geq 90 \%$ 
Morbidity and Mortality Weekly Report

TABLE 1. Vaccination coverage, by vaccine and World Health Organization (WHO) region — worldwide, 2015

\begin{tabular}{|c|c|c|c|c|c|c|c|c|}
\hline \multirow[b]{2}{*}{ Vaccine } & \multirow{2}{*}{$\begin{array}{c}\text { No. countries } \\
\text { including vaccine* }\end{array}$} & \multicolumn{7}{|c|}{ WHO region $(\% \text { coverage })^{\dagger}$} \\
\hline & & Global & African & Americas & Eastern Mediterranean & European & South-East Asia & Western Pacific \\
\hline BCG & 158 & 88 & 80 & 95 & 87 & 89 & 87 & 96 \\
\hline HepB BD & 97 & 39 & 10 & 72 & 23 & 39 & 34 & 83 \\
\hline НерВ3 & 185 & 84 & 76 & 89 & 80 & 81 & 87 & 90 \\
\hline DTP3 & 194 & 86 & 76 & 91 & 80 & 93 & 87 & 94 \\
\hline Hib3 & 191 & 64 & 76 & 90 & 80 & 77 & 56 & 25 \\
\hline Pol3 & 194 & 86 & 76 & 91 & 80 & 94 & 86 & 96 \\
\hline Rota & 84 & 23 & 41 & 76 & 21 & 18 & 0 & 1 \\
\hline PCV3 & 129 & 37 & 59 & 85 & 48 & 48 & 5 & 11 \\
\hline MCV1 & 194 & 85 & 74 & 94 & 76 & 94 & 85 & 96 \\
\hline RCV1 & 147 & 46 & 12 & 94 & 45 & 94 & 14 & 89 \\
\hline MCV2 & 160 & 61 & 18 & 53 & 68 & 89 & 71 & 93 \\
\hline
\end{tabular}

Abbreviations: $B C G=$ Bacille Calmette-Guérin vaccine; DTP3 $=3$ doses of diphtheria-tetanus-pertussis vaccine; HepB BD $=$ birth dose of hepatitis B vaccine; HepB3 = 3 doses of hepatitis B vaccine; $\mathrm{Hib3}=3$ doses of Haemophilus influenzae type b vaccine; $\mathrm{MCV} 1=$ first dose of measles-containing vaccine $(M C V) ; M C V 2=$ second dose of MCV; PCV3 = 3 doses of pneumococcal conjugate vaccine; Pol3 = 3 doses of polio vaccine; RCV1 = first dose of rubella-containing vaccine; Rota = final dose of rotavirus vaccine.

* Number of countries that include the specified vaccine in the routine immunization schedule.

+ Weighted regional average. Coverage based on all countries listed in region or globally, including those that have not yet introduced a given vaccine.

national MCV1 coverage target, ${ }^{* *}$ including 51 (91\%) of 56 high-income countries and five (16\%) of 31 low-income countries (Table 2).

During 2010-2015, global coverage increased for completed series of rotavirus (from $8 \%$ to $23 \%$ ), PCV (11\% to $37 \%$ ), rubella (35\% to $46 \%)$, Hib ( $42 \%$ to $64 \%)$ and $\mathrm{HepB}(74 \%$ to $84 \%$ ) vaccines (Table 1 )]. For the universally recommended vaccines that have been introduced globally into national schedules of most $(>80 \%)$ countries, coverage estimates in low-income countries were lower than those in higher-income countries (Table 2). Among the 50 lower middle-income countries, a higher percentage of the 13 non-Gavi-eligible lower middle-income countries had achieved $\geq 90 \%$ national vaccination coverage than had the 37 Gavi-eligible lower middle-income countries for MCV1 (54\% in non-Gavi eligible versus 38\% in Gavi-eligible lower middle-income countries), MCV2 (31\% versus 24\%), DTP3 (46\% versus 43\%), Pol3 (54\% versus $43 \%$ ), HepB3 (46\% versus $43 \%$ ), and Hib3 ( $46 \%$ versus $43 \%)$.

\section{Discussion}

Commitment by country governments and the collaboration of multiple global immunization partners has led to a reduction in the number of incompletely vaccinated children to an all-time low of 19.4 million in 2015 . Since 2000, approximately 70 low- and middle-income countries have benefitted from Gavi support to increase equitable use of vaccines by strengthening health systems and accelerating

\footnotetext{
** The 2015 milestone in the Global Measles and Rubella Strategic Plan 2012-2020 was to achieve $\geq 90 \%$ coverage with the first routine dose of MCV (or measles-rubella-containing vaccine as appropriate) nationally and $>80 \%$ vaccination coverage in every district or equivalent administrative unit. http:// measlesrubellainitiative.org/wp-content/uploads/2013/06/Measles-RubellaStrategic-Plan.pdf.
}

the introduction and use of newer and underused vaccines such as PCV and rotavirus and rubella vaccines. However, despite substantial progress in increasing the number of children vaccinated worldwide, global DTP3, MCV1 and Pol3 coverage has remained at $84 \%-86 \%$ since 2010 , with coverage improving in some countries and deteriorating in others; $68(35 \%)$ countries have not yet met the Global Vaccine Action Plan 2011-2020 target of 90\% national DTP3 coverage. Coverage varies widely among and within WHO Regions, countries, and communities. Even though vaccines are being introduced into low-income countries more rapidly than ever before (8), equity in coverage has not yet been achieved, and disparities exist, even for vaccines with long-standing recommendations, such as DTP and MCV, with higher coverage in higher-income countries. Furthermore, in lower middle-income countries, vaccination coverage in Gavi-eligible countries still lags behind coverage in non-Gavi-eligible countries despite Gavi support, and nine of the 10 countries with the largest number of incompletely vaccinated children are current or former Gavi-eligible countries, indicating that continued Gavi support for immunization system strengthening is necessary. Ensuring that eligible persons within each district are fully vaccinated according to the national schedule regardless of location, age, sex, ethnicity, educational level, and socioeconomic level is a considerable challenge to the successful delivery of equitable immunization services.

WHO/UNICEF vaccination coverage estimates are supported by or based on vaccination coverage surveys that disaggregate coverage data by demographic and other factors. The Demographic and Health Surveys and Multiple Indicator Cluster Surveys conducted during 2005-2013 indicate that DTP3 coverage is lower in children from poorer households 
TABLE 2. Number of countries reaching national $90 \%$ coverage with MCV1, MCV2, DTP3, Pol3, HepB3, and Hib3 vaccines, by World Bank income category - worldwide, 2015

\begin{tabular}{|c|c|c|c|c|c|c|}
\hline \multirow[b]{2}{*}{ Income category* (no. countries) } & \multicolumn{6}{|c|}{ Vaccines with $90 \%$ coverage, no. (\%) of countries } \\
\hline & MCV1 & MCV2 & DTP3 & Pol3 & НерВ3 & Hib3 \\
\hline High (57) & $52(91)$ & $34(60)$ & $56(98)$ & $54(95)$ & $43(76)$ & $55(96)$ \\
\hline Upper-Middle (54) & $40(73)$ & $27(50)$ & $38(70)$ & $35(65)$ & $39(72)$ & $30(56)$ \\
\hline Lower-Middle (50) & $21(42)$ & $13(26)$ & $22(44)$ & $23(46)$ & $22(44)$ & $22(44)$ \\
\hline Low (31) & $5(16)$ & $1(3)$ & $8(26)$ & $8(26)$ & $8(26)$ & $8(26)$ \\
\hline All income categories (192) & $117(61)$ & $75(39)$ & $124(65)$ & $120(63)$ & $113(57)$ & $115(60)$ \\
\hline
\end{tabular}

Abbreviations: $M C V 1$ = first dose of measles-containing vaccine (MCV); MCV2 = second dose of MCV; DTP3 = 3 doses of diphtheria-tetanus-pertussis vaccine; Pol3 = 3 doses of polio vaccine; HepB3 $=3$ doses of hepatitis B vaccine; Hib3 $=3$ doses of Haemophilus influenzae type $b$ vaccine.

* World Bank income category based on countries' per capita gross national income (GNI) in 2015. Argentina was categorized based on 2014 GNI per capita. Cook Islands and Niue are not included because of lack of recently available GNI estimates.

FIGURE. Average 3-dose diphtheria-tetanus-pertussis vaccine (DTP3) coverage and estimated number of incompletely vaccinated children, by country income category* -10 countries $^{\dagger}$ and worldwide, 2015

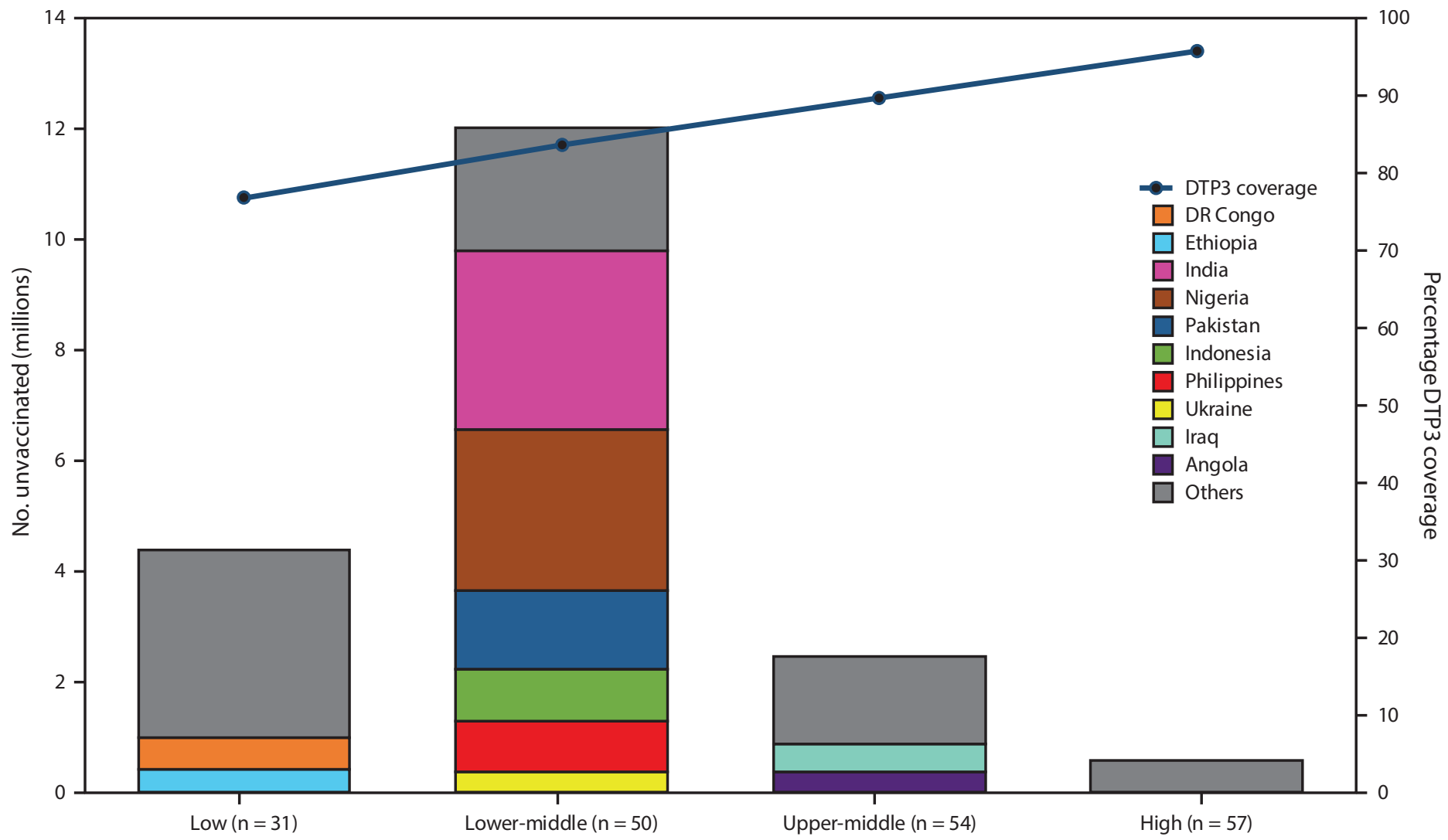

Income classification

Abbreviation: DR Congo = Democratic Republic of the Congo.

* Categories are 2016 World Bank income classifications, which are based on 2015 per capita gross national income and eligibility for financial support from Gavi, the Vaccine Alliance for new vaccine introduction at any time since 2000. Argentina was categorized based on 2014 GNI per capita and Cook Islands and Niue are not included because of lack of World Bank estimate for country income category.

† The 10 countries specified are those with the largest number of incompletely vaccinated children. Others = all other countries within given country income category.

and increases with higher economic status in most low- and middle-income countries (9). These surveys also indicate that coverage is generally higher in urban than rural areas and increases with level of mother's education; no significant differences in coverage are observed by sex.
The findings in this report are subject to at least two limitations. First, inaccuracy in estimation of target population figures might result in over- or under-estimation of administrative coverage. Second, parental recall bias could lead to inaccurate estimates of survey-based coverage $(2,3)$. 


\section{Summary}

What is already known about this topic?

In 1974, the World Health Organization (WHO) established the Expanded Program on Immunization to ensure that all children have access to routinely recommended vaccines. Since then, global coverage with vaccines to prevent tuberculosis, diphtheria, tetanus, pertussis, poliomyelitis, and measles has increased from $<5 \%$ to $\geq 85 \%$, and additional vaccines have been added to the recommended schedule. Coverage with the third dose of diphtheria-tetanus-pertussis vaccine (DTP3) by age 12 months is an indicator of immunization program performance.

What is added by this report?

Global DTP3 coverage has not increased above $85 \%-86 \%$ since 2010. Vaccination coverage varies widely across WHO Regions, countries, and districts, and between population groups and communities, with lower coverage and higher numbers of under-immunized children in lower-income countries and among children from poorer households.

What are the implications for public health practice?

Equitable access to immunization to achieve and sustain high coverage and reduce child mortality can be enhanced through continued financial and technical support for program strengthening and vaccine introductions in lower-income settings; community engagement to increase vaccination acceptance and demand; the collection and use of high quality vaccination data; and government commitment to initiatives to improve immunization services.

Equitable access to immunization is a key goal of the Global Vaccine Action Plan 2011-2020 and is essential to reducing child mortality. Equity-oriented immunization programs that include effective community-centered activities to communicate the value and benefits of vaccination are required to reach marginalized populations and sustain high coverage. Because disease burden is often disproportionately higher in disadvantaged populations, reaching these persons could also have a larger health impact and contribute to economic development (6). National commitment and ownership of initiatives to introduce new vaccines and achieve high vaccination coverage is essential to the success of such programs.

Although global commitment and Gavi support have improved introduction of new vaccines into low-income countries (8), wide disparities in vaccination coverage by country income group exist, even for vaccines recommended since the inception of the Expanded Program on Immunization. The proportion of lower middle-income countries reaching the Global Vaccine Action Plan 2011-2020 targets of 90\% coverage could be improved for both Gavi-eligible and non-Gavi-eligible countries, reflecting that resources are still needed to provide adequate system strengthening even after vaccines have been introduced. Strengthening immunization services, especially in countries with the highest numbers of undervaccinated children, should be a priority to help achieve the United Nation's third Sustainable Development Goal of ending preventable deaths of newborns and children aged $<5$ years by $2030(10)$.

\footnotetext{
${ }^{1}$ Epidemic Intelligence Service, CDC; ${ }^{2}$ Global Immunization Division, CDC; ${ }^{3}$ Department of Immunization, Vaccines and Biologicals, World Health Organization; ${ }^{4}$ Division of Data, Research and Policy, United Nations Children's Fund.

Corresponding author: Rebecca Casey, rcasey@cdc.gov, 404-498-1474.
}

\section{References}

1. Subaiya S, Dumolard L, Lydon P, Gacic-Dobo M, Eggers R, Conklin L. Global routine vaccination coverage, 2014. MMWR Morb Mortal Wkly Rep 2015;64:1252-5. http://dx.doi.org/10.15585/mmwr.mm6444a5

2. Burton A, Monasch R, Lautenbach B, et al. WHO and UNICEF estimates of national infant immunization coverage: methods and processes. Bull World Health Organ 2009;87:535-41. http://dx.doi.org/10.2471/BLT.08.053819

3. World Health Organization. Immunization, vaccines and biologicals — data, statistics and graphs. Geneva, Switzerland: World Health Organization; 2016. http://www.who.int/immunization/monitoring_surveillance/en

4. The World Bank. World Bankcountryandlendinggroups. New York, NY:The World Bank; 2016. https://datahelpdesk.worldbank.org/knowledgebase/ articles/906519-world-bank-country-and-lending-groups

5. Gavi, the Vaccine Alliance. Countries eligible for support. Geneva, Switzerland: Gavi, the Vaccine Alliance; 2015. http://www.gavi.org/ support/sustainability/countries-eligible-for-support/

6. World Health Organization. Global vaccine action plan 2011-2020. Geneva, Switzerland: World Health Organization; 2013. http://www. who.int/immunization/global_vaccine_action_plan/en/

7. Glatman-Freedman A, Nichols K. The effect of social determinants on immunization programs. Hum Vaccin Immunother 2012;8:293-301. http://dx.doi.org/10.4161/hv.19003

8. Loharikar A, Dumolard L, Chu S, Hyde T, Goodman T, Mantel C. Status of new vaccine introduction-worldwide, September 2016. MMWR Morb Mortal Wkly Rep 2016;65:1136-40. http://dx.doi.org/10.15585/ mmwr.mm6541a3

9. World Health Organization. State of inequality: reproductive, maternal, neonatal and child health. Geneva, Switzerland: World Health Organization; 2015. http://www.who.int/gho/health_equity/report_2015/en/

10. United Nations. Transforming our world: the 2030 agenda for sustainable development. New York, NY: United Nations. https:// sustainabledevelopment.un.org/content/documents/21252030\%20 Agenda\%20for\%20Sustainable\%20Development $\% 20$ web.pdf 